\title{
Determinantes del precio de los esclavos en el caribe neogranadino en el siglo XVIII
}

\author{
Determinants of slaves price in the neogranadino \\ Caribbean in the XVIII century
}

\author{
Adolfo Meisel Roca \\ Maestro y doctor en Economía de la Universidad de Illinois \\ Rector de la Universidad del Norte, Colombia \\ https://orcid.org/0000-0003-1255-9867 \\ Ángela Granger Serrano \\ Economista y asistente de investigación de la Universidad del Norte, Colombia \\ https://orcid.org/0000-0001-6108-9625 \\ agranger@uninorte.edu.co \\ Fecha de recepción: 4 de diciembre del 2018 \\ Fecha de aceptación: 11 de enero del 2019 \\ Disponible en línea: 18 de enero del 2019
}

Sugerencia de citación: Meisel Roca, A. y Granger Serrano, A. (2019). Determinantes del precio de los esclavos en el caribe neogranadino en el siglo XVIII. tiempo\&economía,

\section{RESUMEN}

En este trabajo presentamos un modelo económico sencillo para explicar el precio de los esclavos en el Caribe neogranadino en el siglo xvIII y comienzos del xIx. En este, el precio se explica por la edad, la edad al cuadrado, el género, si era calificado o no, si trabajaban en las minas, y si estaba sano o no. La base de datos, que se construye usando información del Archivo

* Los autores agradecen los comentarios de Diana Ricciulli y Julio Romero, así como el apoyo para esta investigación de Juliana Gamboa. 
General de la Nación, incluye precios para un total de 1.064 personas que trabajaban en las haciendas, minas y tejares de la región caribeña neogranadina.

Palabras clave: precios, esclavos, Caribe, Nueva granada, Colonia, Mínimos Cuadrados Ordinarios, historia económica

Códigos JEL: B15, D40, N36, N56

\section{ABSTRACT}

In this paper, a simple model is estimated through ordinary least square to explain the price of slaves in the Caribbean region of the Viceroyalty of New Granada in the 18th century and first decade of the 19th century. The price is explained by age, age squared, gender, degree of qualification, if the person worked in a mine, and if the slave was sick or in good health. The statistical data was obtained from the Archivo General de la Nación in Bogotá, Colombia, and it includes 1.064 persons who worked in haciendas, mines and tile workshops.

Keywords: prices, slaves, Caribbean region, New Granada, Colony, Ordinary Least Square, Economic history.

JEL Codes: B15, D40, N36, N56 


\section{Introducción}

La Costa Caribe colombiana es una región que tiene una extensión de 134.135 kilómetros cuadrados y alberga en sus siete departamentos el 21,0\% de la población del país. Desde el siglo xvII la esclavitud fue fundamental para las actividades productivas, tanto en las zonas urbanas como en las rurales; y fue especialmente importante en el Puerto de Cartagena, pero también en los puertos fluviales como Mompox y Lorica. Las consecuencias económicas y sociales de largo plazo de dicha institución se manifiestan en la actualidad en las enormes desigualdades económicas y sociales que se observan en la región caribeña, y en los bajos índices de desarrollo humano de buena parte de su población. Por esa razón, el estudio de la esclavitud colonial en esta región es de gran relevancia.

En el periodo colonial la costa caribeña estaba compuesta por las provincias de Cartagena y Santa Marta, y el territorio de la Guajira (Mapa 1). En este artículo presentamos los resultados de un análisis econométrico del precio de los esclavos, al estilo de los realizados por la Nueva Historia Económica norteamericana. Para ello construimos una base de datos con 1.064 observaciones del precio de los esclavos y de las características de esas personas en cuanto a edad, género, estado de salud, ocupación y calificación laboral. Toda la información se recopiló en la Sección Colonia del Archivo General de la Nación.

Tal como veremos, hay muchos estudios econométricos sobre el precio de los esclavos en América, tanto para Estados Unidos, como para el Caribe insular y las colonias españolas. Sin embargo, para el caso de Colombia a la fecha no hay un solo estudio que aplique los métodos de la Nueva Historia Económica para el análisis estadístico de dichos precios.

A continuación, se presenta un balance sintético de los estudios económicos sobre la esclavitud en relación a su rentabilidad y a los determinantes del precio de los esclavos. En la siguiente sección describimos las características principales de la esclavitud en el Caribe neogranadino durante el siglo xVIII. Luego, se desarrolla un análisis estadístico del precio de los esclavos en el Caribe neogranadino con información referida al siglo xVIII y los primeros años del siglo xIx. En la última sección presentamos las conclusiones.

\section{Revisión de la literatura}

La esclavitud no sólo ha sido una institución juzgada social y moralmente, sino que también desde un punto de vista económico se ha evaluado su estructura, eficiencia y rentabilidad. Para la experiencia de Estados Unidos, durante el siglo xx los historiadores han discutido ampliamente las características económicas de esa institución, especialmente acerca de su eficiencia, rentabilidad y sostenibilidad económica en el largo plazo. En un balance historioGráfico muy detallado sobre los aportes de la Nueva Historia Económica o Cliometría a los estudios sobre la esclavitud en Estados Unidos, Richard C. Sutch (2018) resaltó los trabajos de Conrad y Meyer (1958), los estudios acerca de qué tan auto suficientes fueron las plantaciones esclavistas del Sur, el debate sobre la reproducción de los esclavos y hasta qué punto fue promovida deliberadamente, y así también, las controversias que causó el libro Time on the Cross de Fogel y Engerman (1974). 


\section{Mapa 1. El Caribe neogranadino en el siglo XVIII}

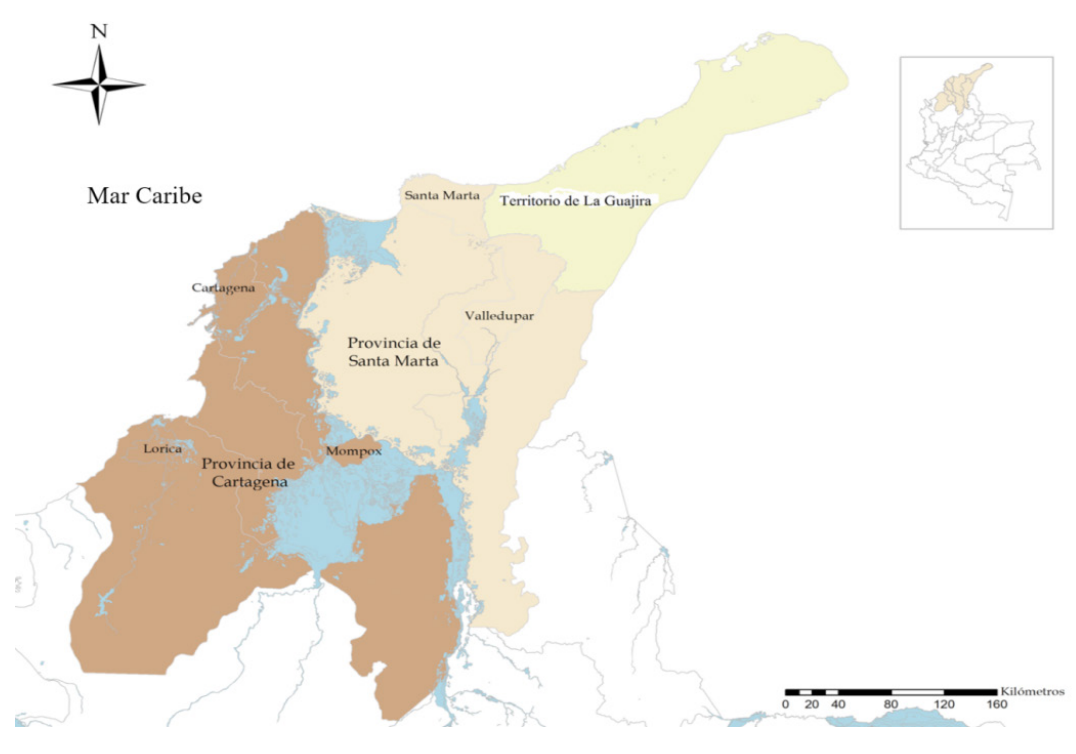

Ramsdell (1929) sostuvo que, en el caso del Sur de Estados Unidos, el sistema llegó a su límite en ganancias y en tierras alrededor de 1860. Según Phillips (1905), a pesar de la disciplina los trabajadores negros eran ineficientes y, en general, la esclavitud fue un obstáculo para el progreso del Sur, privando a la región de la ventaja natural que el monopolio del algodón le debió haber producido. Asimismo, hay quienes aseveran que la Guerra Civil entre el Norte y el Sur de Estados Unidos fue innecesaria, y que el alto precio que se pagó por ella en vidas y los años de pobreza de la posguerra, pudieron haberse evitado "esperando un poco hasta ver caer la esclavitud por su propio peso." (Ramsdell, 1929).

Sin embargo, esta visión no fue muy respaldada; por ejemplo, los historiadores económicos Conrad y Meyer (1958) argumentaron que la economía esclavista antes de la Guerra Civil fue eficiente tanto para las tierras más productivas del Sur de los Estados Unidos, conocidas como el cinturón del algodón, como para las menos productivas, que estaban dedicadas a la crianza y comercialización de esclavos. Esos autores analizaron la esclavitud norteamericana a través de dos funciones de producción que describen dos actividades distintas: la producción agrícola y la producción de esclavos. En el primer caso el producto es un bien final; y en el segundo, un bien intermedio. Partiendo de este enfoque, se estima la tasa de rendimiento sobre el precio de compra de los esclavos, que debió ser al menos igual a la tasa de interés disponible en los mercados de capital durante el periodo evaluado, 1830-1860.

Conrad y Meyer (1958) realizan un análisis del costo de los esclavos dedicados a las labores de campo y los ingresos esperados de dicha inversión. Los resultados apoyan la conclusión de que la esclavitud fue rentable para todo el Sur y, en consecuencia, no había nada económicamente autodestructivo en esa economía y su abolición requería algo más que el funcionamiento de "fuerzas económicas inexorables".

El análisis económico de la esclavitud se ha hecho también para otras zonas del continente americano. En el caso colombiano, Sharp (1975) utilizó la información consignada en inventarios, registros y censos del Archivo General de la Nación, para examinar la rentabilidad de la 
esclavitud minera en el Chocó entre 1680-1810. El autor argumentó que la esclavitud fue una actividad lucrativa durante la primera mitad del siglo xvIII, alcanzando una rentabilidad promedio del $32,0 \%$ en 1724 y $12,7 \%$ en 1759 , ambas por encima del $5 \%$ de interés que producía la mejor inversión segura alternativa, el prestar dinero al interés. En consecuencia, los propietarios de minas, atraídos por las altas utilidades, reinvirtieron en la compra de más esclavos, duplicando su número entre 1759 y 1782. Esta situación llevó a un aumento de los trabajadores, lo cual implicó un declive de las ganancias mineras en las décadas siguientes. Finalmente, Sharp (1975) asegura que "a pesar de que la esclavitud en el Chocó fue temporalmente una institución engorrosa e ineficiente, en el siglo xvIII no estuvo nunca en peligro de autodestruirse", lo cual está en línea con lo encontrado por Conrad y Meyer (1958) para los Estados Unidos.

Ahora bien, el análisis de rentabilidad es sólo una de las líneas de investigación en torno a la esclavitud. El comportamiento y los determinantes de los precios de los esclavos también ha sido objeto de muchos estudios, en tanto el análisis de la evolución, estructura y determinantes de los precios de los esclavos permite una mejor comprensión del funcionamiento del sistema esclavista.

En la historiografía económica se ha evaluado el efecto de algunas variables en el precio de los esclavos, por ejemplo, el género se ha tenido en cuenta en algunas investigaciones. En el Perú, especialmente en la esclavitud minera, el precio de las mujeres era en promedio un $18 \%$ inferior al de los hombres (Newland y San Segundo, 1996). En Cuba se empleaba una gran proporción de esclavos en las plantaciones azucareras donde la fuerza física era crítica, por lo que el precio de las esclavas podía ser entre un $90 \%$ y un $95 \%$ del precio de un esclavo masculino con iguales condiciones (Moreno et ál., 1983). En contraste, en La Plata, Uruguay, no se registraron diferencias significativas entre esclavos hombres y mujeres (Newland y San Segundo, 1996). Asimismo, se encontró que el precio de las esclavas con hijos tenía una prima, que aumentaba con la edad del niño (Kotlikoff, 1978; Chenny et ál., 2003).

En un estudio para Santiago de Chile, Cussen et ál. (2016) realizaron un análisis de regresión para establecer la relación entre el precio de los esclavos y sus características observables: edad, género, raza, origen y salud. La información se tomó de 3.851 operaciones de venta entre 1773 y 1822 con 4.556 esclavos, disponibles en el Archivo Histórico Nacional de Chile. Los resultados indican que el precio de las esclavas era 26\% mayor al de los esclavos hombres y, a su vez, los esclavos africanos (bozales) eran más costosos que los criollos. Si bien, ambos resultados son contrarios a la tendencia general, el primero es consecuencia de una mayor demanda de mujeres para las ocupaciones domésticas, que eran las que predominaban en el mercado urbano de Santiago. La explicación para el alto precio relativo de los africanos no es clara; los autores sugieren dos posibles explicaciones: primero, una preferencia de los propietarios por esclavos bozales, pues se percibían más dependientes de sus dueños y por tanto más sumisos; y segundo, un posible aumento en los precios de los esclavos africanos en Santiago de Chile a causa de la alta demanda por parte del mercado peruano.

Contrario a los resultados de Cussen et ál. (2016), generalmente se argumenta que los esclavos criollos eran más costosos que los africanos, dada su mayor resistencia ante las enfermedades y su adaptación a las condiciones climáticas y culturales locales. Respecto a otras características, se encontró también que los mulatos tenían un precio más bajo que los demás (Cussen et ál., 2016; Newland y San Segundo, 1996). 
Por otra parte, e independientemente de las particularidades de cada región, hay un consenso en cuanto a la existencia de una relación en forma de $U$ invertida entre la edad del esclavo y su precio. Esto sugiere un precio ascendente durante la niñez y la juventud, y un descenso a partir de la edad en la que se alcanza el máximo precio. Dicho precio máximo se encontraría entre los 16 y 34 años en Santiago de Chile (Cussen et ál., 2016); entre los 27 y los 29 años en Perú (Newland y San Segundo, 1996); y en Cuba, entre los 25 y 30 años en los esclavos masculinos, y alrededor de 25 años para las mujeres (Moreno et ál., 1983).

Algunos autores utilizan el enfoque del capital humano para la estimación de los determinantes de los precios. Así, en la medida que las transacciones de compra y venta registraban el estado de salud de los esclavos y sus habilidades, se puede establecer el efecto de estas variables en los precios. Acorde con lo obtenido por Newland y San Segundo (1996), las habilidades incrementaban en un $66 \%$ y un $25 \%$ el valor de un esclavo en Perú y La Plata, respectivamente. Así mismo, en la República de Mauricio los esclavos especialmente hábiles tenían un precio $14 \%$ por encima del precio de aquellos dedicados a la agricultura y $17 \%$ mayor al de los que trabajaban en las casas de los propietarios (Chenny et ál., 2003). Al igual que, en Nueva Orleans los artesanos también fueron altamente valorados (Kotlikoff, 1978).

La salud era esencial para establecer el precio de un esclavo. Los esclavos con enfermedades eran más económicos que aquellos que estaban saludables. En La Plata, por ejemplo, tal característica fue más valorada que en Perú; en el primer caso, un esclavo sano costaba $86 \%$ más que uno enfermo, mientras que en Perú esta cifra era del 51\% (Newland \& San Segundo, 1996). En la República de Mauricio, por su parte, los esclavos con discapacidades costaban 56\% menos (Chenny et ál., 2003).

En el análisis de los precios de los esclavos también se tienen en cuenta algunas características diferentes a las sociodemográficas. Por ejemplo, en ocasiones se añade un componente de estacionalidad. Los cultivos de caña de azúcar fueron la base económica de varias de las sociedades esclavistas, incluidas Cuba y la República de Mauricio. En Cuba, los precios de los esclavos se dispararon en la segunda mitad del siglo xIx debido al dinamismo de la demanda laboral como resultado del aumento del precio del azúcar (Bergad, 1987). Así también, en el análisis para la república africana de Mauricio, entre 1825 y 1827, se encontró que el precio de los esclavos aumentaba en el cuarto trimestre del año, que era la temporada de la cosecha azucarera (Chenny et ál., 2003).

Por su parte, Coleman y Hutchinson (2006) examinan el caso de Luisiana y Nueva Orleans entre 1725 y 1820. Estos autores estimaron el efecto de dos políticas públicas en la estructura de precios de los esclavos. La primera fue "Embargo de Jefferson"1 en 1807, que resultó tener un efecto negativo en el precio de los esclavos en Luisiana (sin incluir Nueva Orleans), y positivo en Nueva Orleans. Y la segunda hace referencia a la legislación que prohibió la importación de esclavos en 1808 y que tuvo un impacto positivo en ambas regiones, pero mayor en Nueva Orleans que en el resto del Estado.

1 Fue una legislación propuesta por Thomas Jefferson, presidente de los Estados Unidos 1801-1809, en la que se prohibió a los barcos estadounidenses salir a puertos extranjeros. Esto, como respuesta a los frecuentes obstáculos y confiscaciones de mercancías de los buques estadounidense en puertos británicos y franceses. 
Finalmente, hay que resaltar que el tema de los precios de los esclavos no se ha analizado econométricamente para el caso del territorio de lo que hoy es Colombia. Es decir, que este es el primer trabajo en nuestro medio donde se aplican al tema los métodos de la Cliometría. Para ello estimamos, por medio de mínimos cuadrados ordinarios, un sencillo modelo con los principales determinantes del precio de los esclavos en el Caribe neogranadino en el siglo xVIII.

\section{Características de la esclavitud en el Caribe neogranadino}

La drástica caída de la población indígena en las provincias del Caribe neogranadino después de la conquista española llevó a que desde comienzos del siglo xvıl buena parte, quizá incluso la mayor parte, de la producción agropecuaria se realizara con mano de obra traída del África. Para el siglo xvIII la presencia de una población de libres de todos los colores permitió que una gran parte de la actividad económica rural y urbana se hiciera con la población de mestizos, mulatos, zambos y negros libres. En el Gráfico 1 podemos observar que en el censo de 1778 el 64\% de la población del Caribe neogranadino estaba constituida por los libres de todos los colores, como se denominaba en los censos de la época a ese grupo.

Gráfico 1. Composición de la población en el Caribe neogranadino, 1778

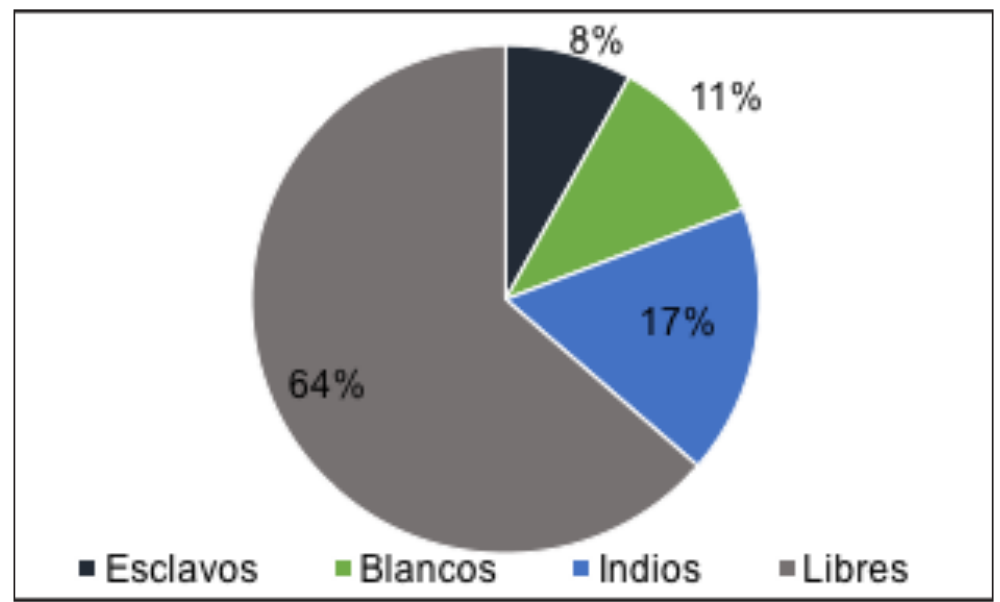

Fuente: cálculos de los autores con datos del Archivo General de la Nación, Mapoteca 7, No. 1353(21); Tovar et ál. (1994).

En el censo de 1778, la población de las provincias de Cartagena, Santa Marta y el territorio de La Guajira, sin contar la población indígena en esa península que los españoles no habían logrado someter, fue de 169.020 (Cuadro 1). La mayoría de la población (70\%) vivía en la provincia de Cartagena, pero toda la región tenía una densidad poblacional muy baja. 
Cuadro 1. Población del Caribe neogranadino, alrededor de 1777-1778

\begin{tabular}{ccc}
\hline & Habitantes & Porcentaje \\
\hline Provincia de Cartagena & 118.403 & $70 \%$ \\
Provincia de Santa Marta & 46.651 & $28 \%$ \\
Territorio de La Guajira & 3.966 & $2 \%$ \\
\hline Total & $\mathbf{1 6 9 . 0 2 0}$ & $\mathbf{1 0 0 \%}$ \\
\hline
\end{tabular}

Fuente: cálculos de los autores con datos del Archivo General de la Nación, Mapoteca 7, No. 1353(21); Tovar et ál. (1994, pp. 507-517).

La composición de la población caribeña de acuerdo con la clasificación usada por las autoridades de la época variaba entre las zonas rural y urbana. En estas últimas casi no había población indígena, 1\%, mientras que en el sector rural el $21,0 \%$ de la población era indígena (Gráfico 2). En contraste, la población blanca y esclava tenía una mayor participación en el área urbana. Los blancos eran el $26 \%$ de la población urbana y solo el $7 \%$ de la rural, y en el caso de los esclavos la participación era del $17 \%$ en las zonas urbanas y del $7 \%$ en las rurales.

Gráfico 2. Composición de la población en el Caribe neogranadino por zona (1778)

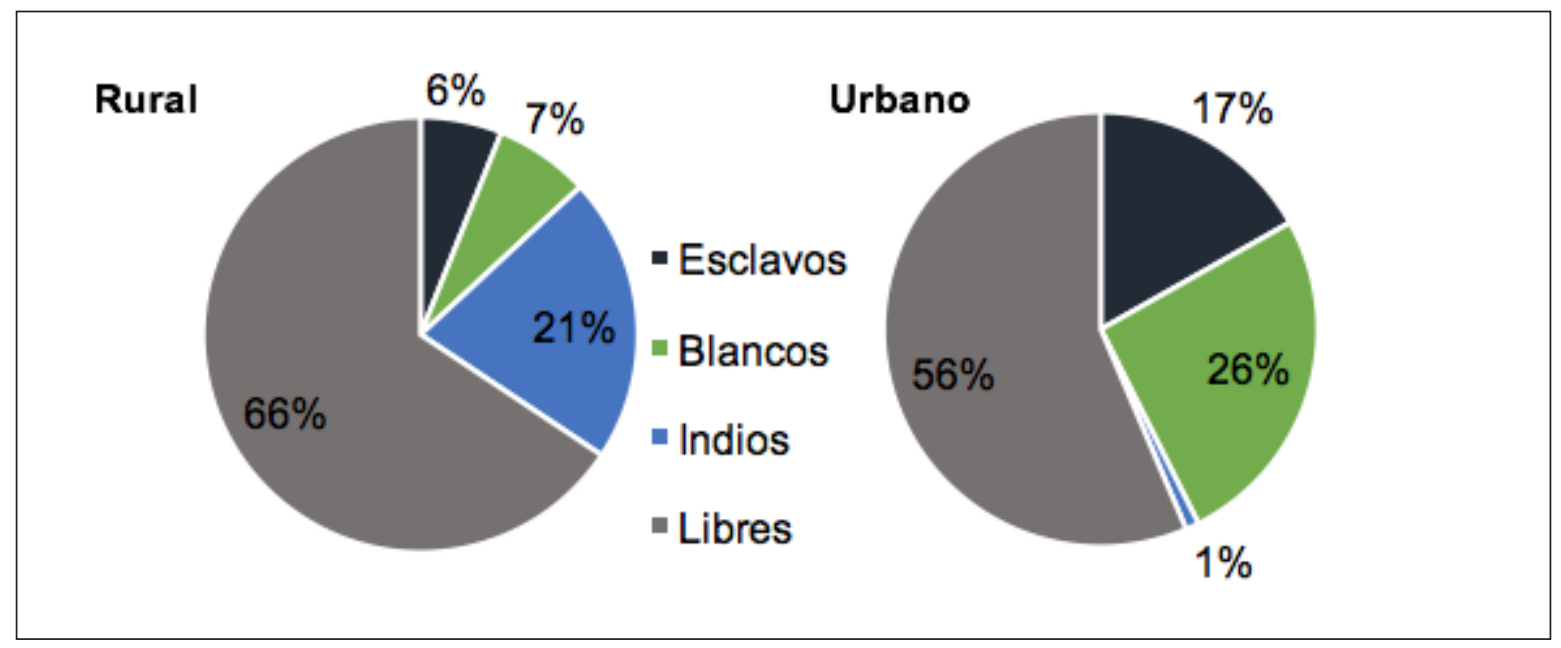

Fuente: cálculos de los autores con datos del Archivo General de la Nación, Mapoteca 7, No. 1353(21); Tovar et ál. (1994).

La principal unidad económica del Caribe insular en el periodo colonial fue la plantación. Estas eran explotaciones agrícolas intensivas en mano de obra esclava y capital. Exportaban casi el $100 \%$ de su producción a Europa. Los productos que se exportaban eran el algodón, el tabaco, pero sobre todo azúcar. En las islas caribeñas las plantaciones podían tener 300 o 400 esclavos, e incluso más. En contraste, en el Caribe colombiano para el periodo colonial las dos explo-

2 Las ciudades que se consideraron fueron Cartagena, Mompox, Ocaña, Lorica, Valledupar, y Santa Marta. Todas ellas tenían una población que era mayor a los 3.500 habitantes. 
taciones agropecuarias con más esclavos que hemos encontrado tenían 100 y 101 esclavos. $^{3}$ La mayor parte de los esclavos rurales del Caribe colombiano estaban dedicados a trabajar en las haciendas ganaderas y en las haciendas trapiche. Estas haciendas no exportaban su producción a los mercados del Caribe o de Europa sino que estaban orientadas a abastecer a los principales centros urbanos del Caribe neogranadino, especialmente Cartagena, que era la ciudad más prospera del virreinato (Meisel, 2016).

Las haciendas ganaderas utilizaban poca mano de obra de manera permanente y los esclavos usualmente estaban destinados a los trabajos que eran menos estacionales u ocasionales. Para estos últimos casos se recurría a menudo a la población de campesinos libres.

Las haciendas trapiches absorbían más mano de obra que las ganaderas. Los trapiches producían mieles y panela para los centros urbanos. Los principales estaban localizados cerca del Canal del Dique, lo cual facilitaba el transporte de las mieles, azúcares y panelas hasta Cartagena.

\section{Análisis econométrico del precio de los esclavos}

Para el estudio de las características de la esclavitud en las llanuras y poblaciones del Caribe neogranadino hemos recopilado información proveniente de varios fondos del Archivo General de la Nación, Sección Colonia. Estos esclavos se encontraban en las haciendas, minas y tejares de las provincias de Cartagena y Santa Marta. El horizonte temporal se extiende desde el siglo xVIII hasta los últimos años de la Colonia, a comienzos del siglo xIx. En total son 2.068 observaciones entre hombres y mujeres. La edad promedio de este grupo es de 31 años y la razón de sexos es de 2,0 (hombres/mujeres). Cabe señalar que algunas haciendas y minas se pueden repetir pues los inventarios donde se establecía el precio se podían realizar en varios años.

Los precios que utilizaremos están todos en pesos de plata de ocho reales, que era la unidad monetaria de las colonias españolas en América por esa época. Como en general hubo estabilidad de precios, el análisis se llevó acabo en términos nominales.

De las 2.068 observaciones de esclavos con las que contamos, sólo en el caso de 1.064 tenemos información acerca de los precios de estos. Esta información de precios se obtiene de los inventarios y avalúos que se hacían periódicamente por parte de los propietarios de haciendas, minas y tejares. Con esas 1.064 observaciones se realizó el análisis econométrico.

Debemos señalar que en el Caribe neogranadino no hubo grandes explotaciones agrarias dedicadas a exportar a los mercados mundiales productos como algodón, tabaco y azúcar, entre otros. Por eso sólo encontramos dos casos de haciendas que tuvieron 100 o más esclavos: San Pablo, 101, y Santa Rosa, 100 (Gráfico 3).

3 Para una discusión sobre las posibles causas por la cual no hubo plantaciones en el Caribe neogranadino ver Abello (2006). 


\section{Gráfico 3. Número de esclavos por hacienda, tejar o mina}

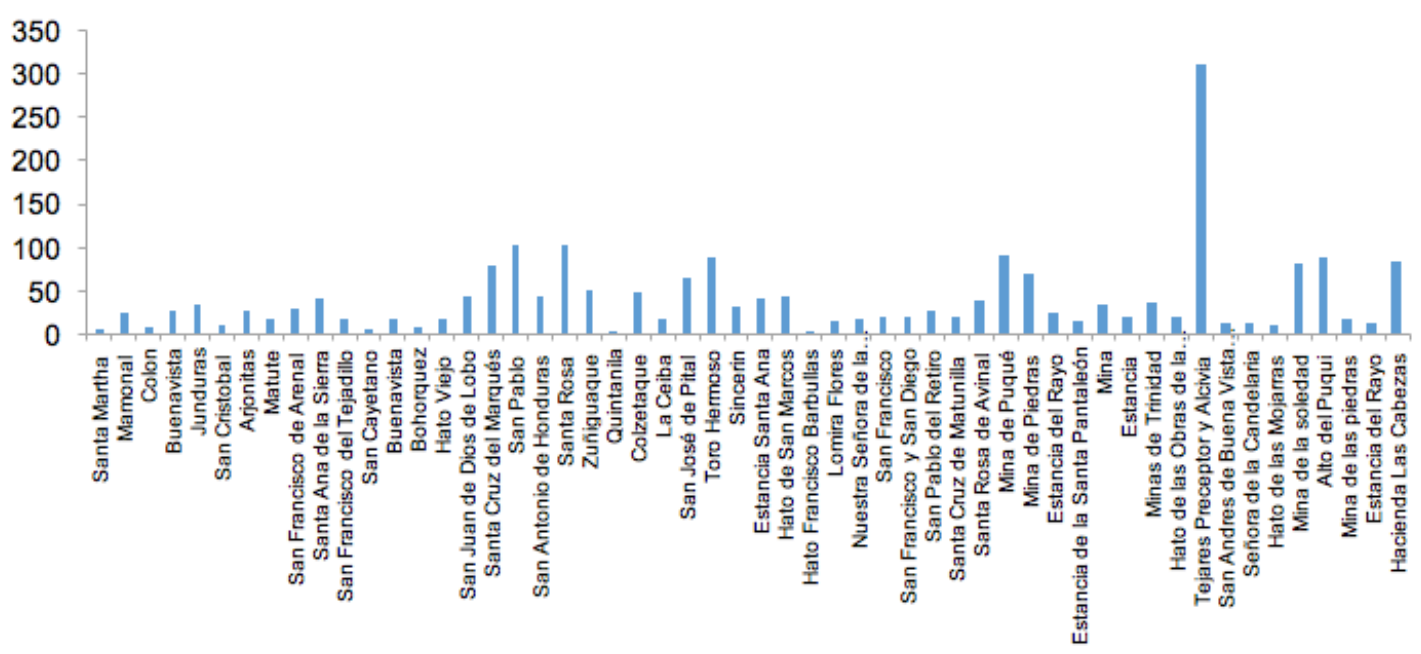

Fuente: Elaboración de los autores con datos de Archivo General de la Nación, Sección Colonia, Temporalidades, Tomo 3, Orden 8, ff. 233-236, 259-260. Sección Colonia, Testamentarias de Bolívar, Tomo 5, Orden 3, ff. 602-610, 612-621, 623-626, 644-645, 682-686, 703-705, 724-725, 732-734. Sección Colonia, Censos Varios Departamentos, ff. 789-790, 799, 800, 804, 817, 823-825, 827,828-832, 838-840. Colombia, Tierras de Bolívar, Tomo I, ff. 137, 924. Tomo II, ff. 636. Tomo III, ff. 396, 399, 402. Tomo V, ff. 452 . Tomo VIII, ff. 75.

Tomo XII, ff. 655. Colonia, Testamentarias de Bolívar, Tomo 3, Folios: 28-31, 39-41, 45-53, 89-99, 118-127, 130-135, $174-179$.

La edad promedio para el grupo de los 1.064 esclavos que estamos estudiando era de 31 años. Es decir, no era una población muy joven. La edad promedio más baja la encontramos en la Hacienda La Ceiba, 18 años, mientras que la más alta se observó en el Hato Barbullas (60 años), pero solo tenía ese único esclavo (Gráfico 4).

Gráfico 4. Edad promedio de los esclavos en haciendas tejares y minas

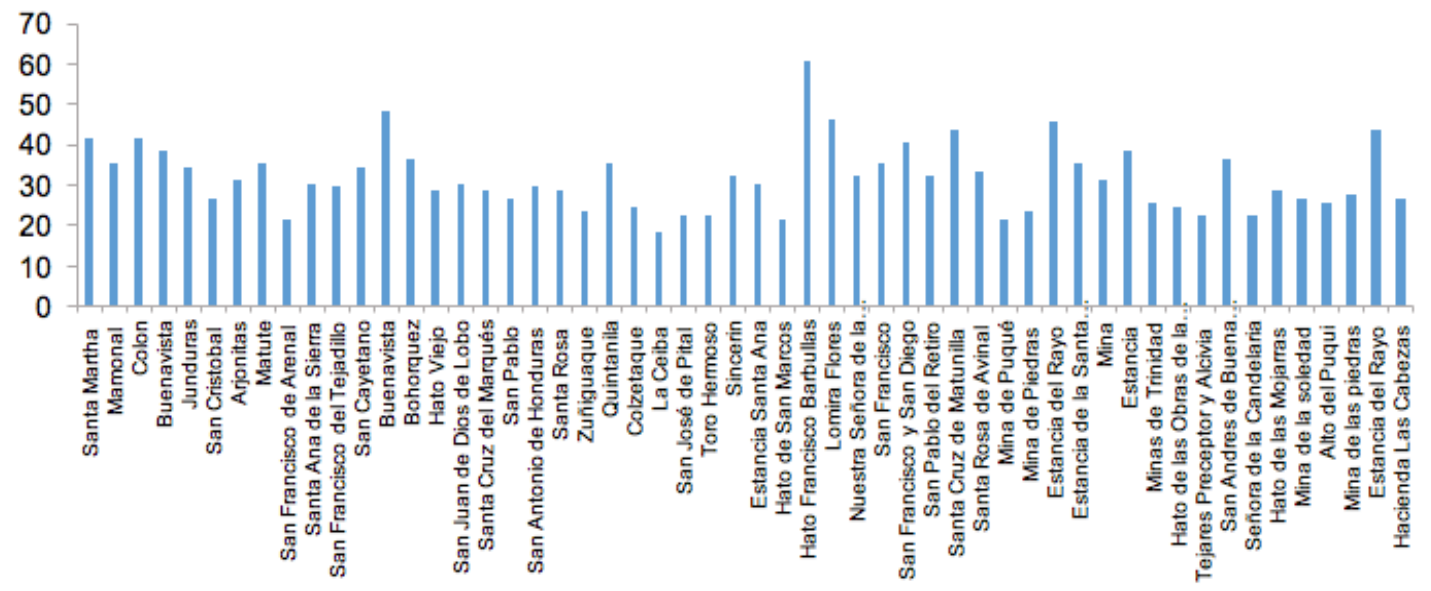

Fuente: Elaboración de los autores con datos de Archivo General de la Nación, Sección Colonia, Temporalidades, Tomo 3, Orden 8 , ff. 233-236, 259-260. Sección Colonia, Testamentarias de Bolívar, Tomo 5, Orden 3, ff. 602-610, 612-621, 623-626, 644-645, 682-686, 703-705, 724-725, 732-734. Sección Colonia, Censos Varios Departamentos, ff. 789-790, 799, 800, 804, 817, 823-825, 827,828-832, 838-840. Colombia, Tierras de Bolívar, Tomo I, ff. 137, 924. Tomo II, ff. 636. Tomo III, ff. 396, 399, 402. Tomo V, ff. 452 . Tomo VIII, ff. 75. Tomo XII, ff. 655. Colonia, Testamentarias de Bolívar, Tomo 3, Folios: 28-31, 39-41, 45-53, 89-99, 118-127, 130-135, $174-179$. 
Una característica de la esclavitud en esta región fue que la razón de sexos, hombres por cada mujer, siempre fue mayor que uno en las zonas rurales. La consecuencia de esto era que la tasa de crecimiento natural de la población esclava era muy baja o negativa y, por lo tanto, dependía para mantenerse o ampliarse de la importación continua de esclavos traídos del África. Aunque en la base de datos de los 2.068 esclavos rurales la razón de sexo era de 2,0, esta variaba bastante de hacienda a hacienda.

Por ejemplo, la Hacienda San Andrés de Buenavista tenía 11 esclavos y había una sola mujer. En contraste, la Hacienda Toro Hermoso con 86 esclavos tenía una razón de sexos de solo 0,25 . En la mayoría de los casos, sin embargo, la razón de sexos tendía a estar solo un poco encima de 1,0 (Gráfico 5).

Gráfico 5. Razón de sexos de los esclavos en haciendas, tejares y minas

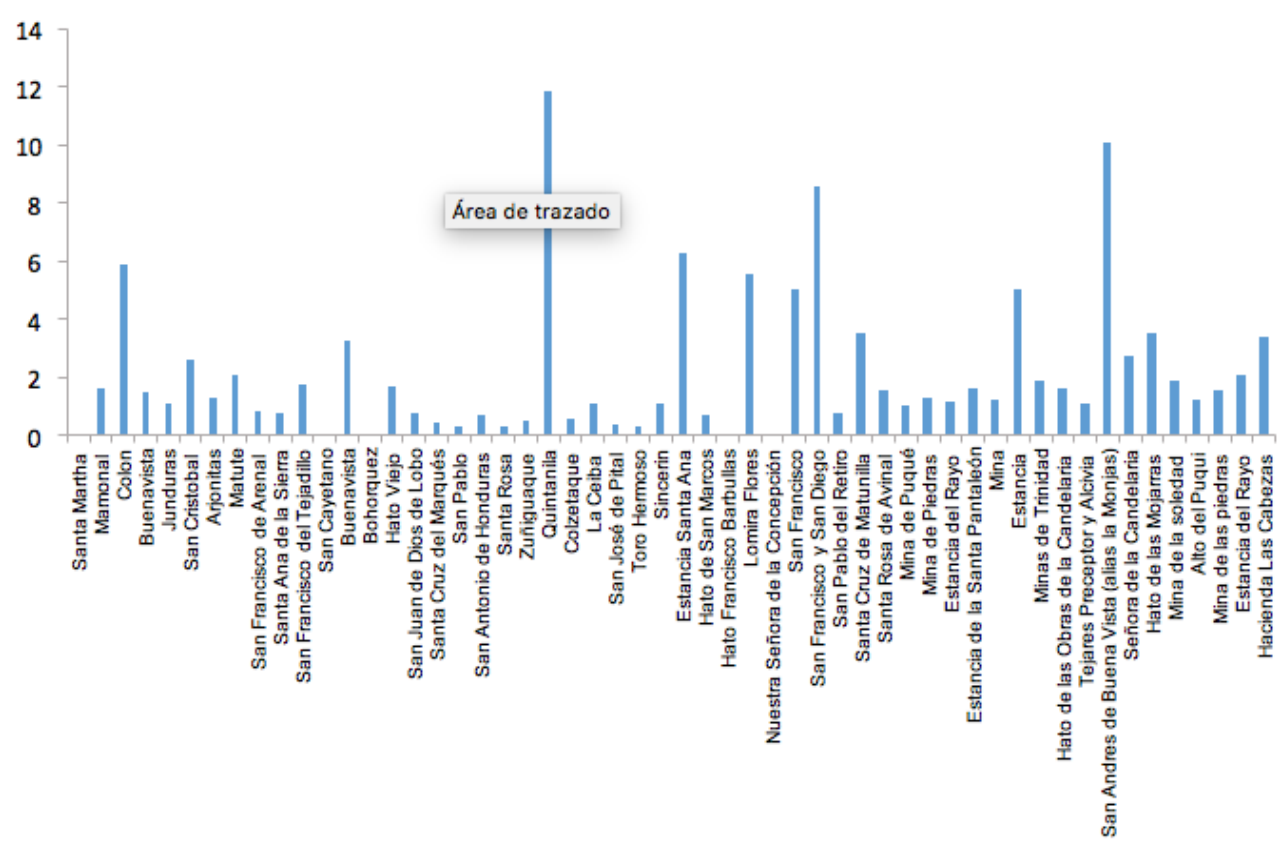

Fuente: elaboración de los autores con datos de Archivo General de la Nación, Sección Colonia, Temporalidades, Tomo 3, Orden 8, ff. 233-236, 259-260. Sección Colonia, Testamentarias de Bolívar, Tomo 5, Orden 3, ff. 602-610, 612-621, 623-626, 644-645, 682-686, 703-705, 724-725, 732-734. Sección Colonia, Censos Varios Departamentos, ff. 789-790, 799, 800, 804, 817, 823-825, 827,828-832, 838-840. Colombia, Tierras de Bolívar, Tomo I, ff. 137, 924. Tomo II, ff. 636. Tomo III, ff. 396, 399, 402. Tomo V, ff. 452 . Tomo VIII, ff. 75. Tomo XII, ff. 655. Colonia, Testamentarias de Bolívar, Tomo 3, Folios: 28-31, 39-41, 45-53, 89-99, 118-127, 130-135, $174-179$.

Otra característica del desbalance de sexos fue que, en las edades de mayor fertilidad, 2034 años, este era aún más acentuado. Así mismo, la pirámide de edades no era con base ancha y rápida disminución hacia la cúspide, como se observa en poblaciones jóvenes con un crecimiento endógeno alto, sino con grupos de edades de tamaño similar como se observa en el Gráfico 6. 


\title{
Gráfico 6. Pirámide poblacional Hacienda Santa Coa, Tejares Preceptor, Alcivia y Tierra Bomba y varias ha- ciendas de la Costa Caribe ${ }^{4}$
}

\author{
$\square \%$ Hombres $\quad$ घ\% Mujeres
}

Edad Promedio: 26 años. Edad Promedio Mujeres: 23 años. Edad Promedio Hombres: 27 años. Razón de sexos: 1,52 Total Esclavos: 1767

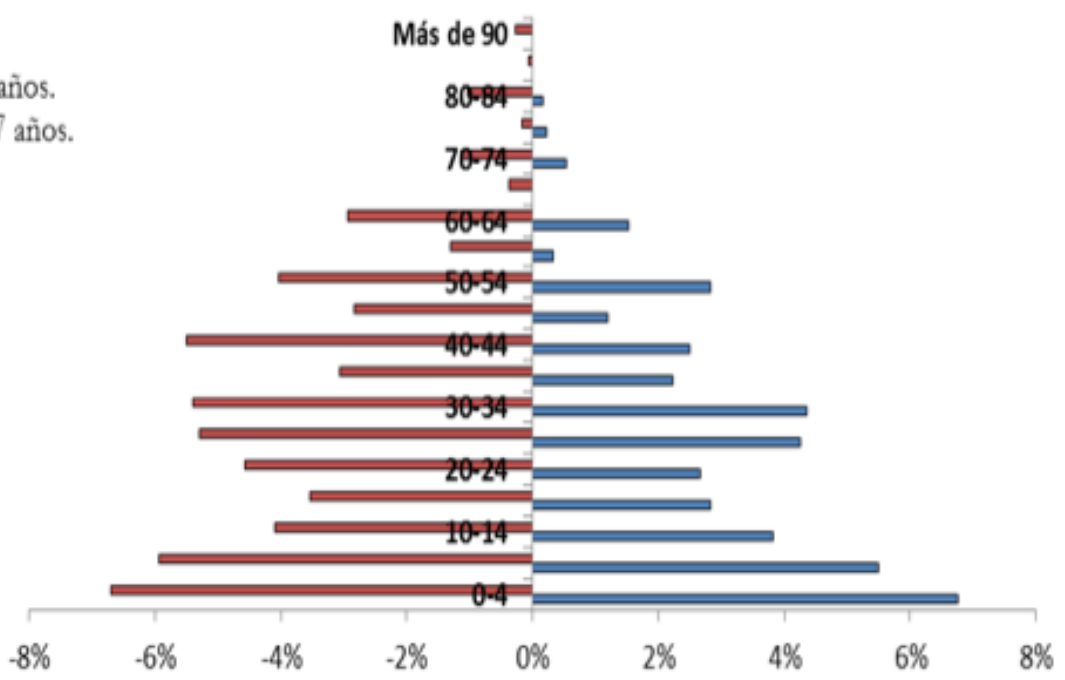

Fuente: Elaboración de los autores con datos de Archivo General de la Nación, Sección Colonia, Temporalidades, Tomo 3, Orden 8, ff. 233-236, 259-260. Sección Colonia, Testamentarias de Bolívar, Tomo 5, Orden 3, ff. 602-610, 612-621, 623-626, 644-645, 682-686, 703-705, 724-725, 732-734. Sección Colonia, Censos Varios Departamentos, ff. 789-790, 799, 800, 804, 817, 823-825, 827,828-832, 838-840. Colombia, Tierras de Bolívar, Tomo I, ff. 137, 924. Tomo II, ff. 636. Tomo III, ff. 396, 399, 402. Tomo V, ff. 452. Tomo VIII, ff. 75.

Tomo XII, ff. 655. Colonia, Testamentarias de Bolívar, Tomo 3, Folios: 28-31, 39-41, 45-53, 89-99, 118-127, 130-135, $174-179$.

Todas estas características de la población esclava se reflejaron también en diferencias en los precios de compra. Un primer acercamiento al precio promedio de los esclavos en el Caribe Neogranadino da cuenta de que las mujeres eran ligeramente más baratas que los hombres, los esclavos enfermos valían menos (cerca de 65 pesos menos en promedio), los esclavos calificados costaban más (cerca de 50 pesos más en promedio), y los esclavos de las minas valían aproximadamente lo mismo que aquellos en otra ocupación. En cuanto a la edad, el rango mejor pagado se registraba entre los 20 y 40 años (Cuadro 1).

El modelo estadístico que utilizamos para un análisis formal del precio de los esclavos en el Caribe neogranadino tiene como variable dependiente el precio del esclavo y como variables independientes la edad, la edad al cuadrado, y las variables dicotómicas, que toman valores de cero o uno, para el estado de la salud ${ }_{1}^{5}$ género, si era calificado o no y si trabajaba en una mina o no:

4 Las haciendas son: Santa Martha, Mamonal, Colón, Buenavista, Junduras, San Cristobal, Arjonitas, Matute, San Francisco de Arenal, Santa Anda de la Sierra, San Francisco del Tejadillo, San Cayetano, Buenavista (labranza), Bohórquez, Hato Viejo, San Juan de Dios de Lobo, Santa Cruz del Marqués, San Pablo, San Antonio de Honduras, Santa Rosa, Zuñiguaque, Quintanilla, Colzetaque, La Ceiba, San José de Pital, Toro Hermoso, Sincerin, Santa Cruz de Matunilla, San Francisco, San Pablo del Retiro, Hato de San Marcos, Estancia Carinca, Estancia Santa Cruz del Paraíso y bienes de Don Cayetano Palacios.

5 Los inventarios del Archivo Nacional especifican las enfermedades del esclavo al momento de la transacción. Esta información se resume en una variable que indica si el esclavo estaba o no enfermo, independiente cual fuere la enfermedad. 


\section{Cuadro 2. Precio promedio de los esclavos}

\begin{tabular}{|c|cc|}
\hline \multirow{2}{*}{ Género } & & Precio promedio \\
& Hombre & 192.9 \\
& Mujer & 172.3 \\
\hline \multirow{2}{*}{ Salud } & Sano & 190.2 \\
& Enfermo & 135.4 \\
\hline \multirow{2}{*}{ Habilidades } & No calificado & 182.3 \\
& Calificado & 233.5 \\
\hline \multirow{2}{*}{ Ocupación } & Otro trabajo & 183.0 \\
& Mina & 184.3 \\
\hline \multirow{3}{*}{ Edad } & $<20$ años & 138.0 \\
& 20-40 años & 264.6 \\
& $40-60$ años & 160.1 \\
& $60-80$ años & 71.4 \\
\hline
\end{tabular}

Fuente: Elaboración de los autores con datos de Archivo General de la Nación, Sección Colonia, Temporalidades, Tomo 3, Orden 8, ff. 233-236, 259-260. Sección Colonia, Testamentarias de Bolívar, Tomo 5, Orden 3, ff. 602-610, 612-621, 623-626, 644-645, 682-686, 703-705, 724-725, 732-734. Sección Colonia, Censos Varios Departamentos, ff. 789-790, 799, 800, 804, 817, 823-825, 827,828-832, 838-840. Colombia, Tierras de Bolívar, Tomo I, ff. 137, 924. Tomo II, ff. 636. Tomo III, ff. 396, 399, 402. Tomo V, ff. 452. Tomo VIII, ff. 75.

Tomo XII, ff. 655. Colonia, Testamentarias de Bolívar, Tomo 3, Folios: 28-31, 39-41, 45-53, 89-99, 118-127, 130-135, $174-179$.

$$
\begin{aligned}
& \text { lnPrecio }=\alpha+\beta E d a d+\gamma E d a d^{2}+\delta E n f e r m o+\mu \text { Género }+ \\
& \rho \text { Califiación }+\sigma \text { Mina }+\varepsilon
\end{aligned}
$$

Se realizó la estimación por mínimos cuadrados ordinarios y todas las variables resultaron significativas al 1,0\%, a excepción de la ocupación en minas. Además, los signos de los coeficientes son los correctos. El $\mathrm{R}^{2}$ ajustado es de 0,72 . Es decir que, con esta ecuación se explica el $72 \%$ de las diferencias en los precios de los esclavos. Estos resultados van acorde a la literatura entorno a los determinantes del precio de los esclavos en otros países de América Latina. En este análisis, el precio de las esclavas se registró $6 \%$ por debajo del precio de los esclavos masculinos (Cuadro 2), similar a los hallazgos para Cuba donde el precio de los hombres era entre $5 \%$ y $10 \%$ superior al de las mujeres. En cuanto a las habilidades y la salud, significaron un precio $15 \%$ y $52 \%$ mayor en el Caribe neogranadino. Sin embargo, estas fueron características igual o más valoradas en otros países como Perú y Uruguay donde los esclavos hábiles se transaban por un precio $66 \%$ y $25 \%$ superior y los esclavos sanos registraban precios $51 \%$ y $86 \%$ más altos, respectivamente. Finalmente, en lo que respecta a la edad, el precio más alto en el Caribe Neogranadino se pagaba por los esclavos de aproximadamente 33 años, mientras que, en Perú y Cuba el precio máximo se pagó por esclavos entre los 25 y 30 años (Newland y San Segundo, 1996; Moreno et ál., 1983). 
Cuadro 3. Determinantes del precio de los esclavos

\begin{tabular}{|c|c|}
\hline Variable & Coeficiente \\
\hline \multirow[t]{2}{*}{ edad } & $0.0910^{* * *}$ \\
\hline & $(0.00182)$ \\
\hline \multirow[t]{2}{*}{ edad2 } & $-0.00139 * * *$ \\
\hline & $(2.88 \mathrm{e}-05)$ \\
\hline \multirow[t]{2}{*}{ enfermo } & $-0.416^{* * *}$ \\
\hline & $(0.0362)$ \\
\hline \multirow[t]{2}{*}{ sexo $($ mujer=1) } & $-0.0619^{* * *}$ \\
\hline & $(0.0220)$ \\
\hline \multirow[t]{2}{*}{ calificación (si=1) } & $0.136^{* *}$ \\
\hline & $(0.0597)$ \\
\hline \multirow[t]{2}{*}{ mina } & 0.0206 \\
\hline & $(0.0230)$ \\
\hline \multirow[t]{2}{*}{ Constant } & $4.186^{* * *}$ \\
\hline & $(0.0271)$ \\
\hline Observations & 991 \\
\hline R-squared & 0.726 \\
\hline
\end{tabular}

\section{Conclusiones}

La esclavitud fue una de las instituciones económicas y sociales que más significó para la actividad productiva en el Virreinato de la Nueva Granada. Gran parte de la actividad de la minería de oro, principal y casi único producto de exportación, se realizaba con el trabajo de los esclavos traídos del África o sus descendientes. Esto era especialmente cierto en el Pacífico, en las tierras bajas de lo que hoy son los departamentos de Chocó, Valle del Cauca, Nariño y el Cauca. Las consecuencias de largo plazo de la esclavitud para esas zonas mineras han sido negativas. En un estudio de Daron Acemoglu, Camilo García-Jimeno y James A. Robinson (2012), se encontró que los municipios del Virreinato de Nueva Granada que en el siglo xvII y xvIII tuvieron esclavitud en la actualidad son más pobres que sus vecinos donde esta fue casi inexistente (Acemoglu et ál., 2012). A pesar de esta impronta negativa, hay una escasez de estudios sobre la historia económica de la esclavitud en el Virreinato de la Nueva Granada, en particular de aquellos que utilizan los métodos cuantitativos y analíticos de la Nueva Historia Económica.

En este trabajo hemos presentado un modelo económico sencillo para explicar el precio de los esclavos en el Caribe neogranadino en el siglo xVIII y comienzos del xIx. En este, el precio se explica por la edad, la edad al cuadrado, el género, si era calificado o no, si trabajaban en las minas, y si estaba sano o no. La base de datos, que se construyó usando información del Archivo General de la Nación, incluye precios para un total de 1.064 personas que trabajaban en las haciendas, minas y tejares de la región caribeña neogranadina. El modelo se estimó por 


\section{Gráfico 7. Relación precio-edad esclavos Hacienda Santa Coa, Tejares Alcivia, Preceptor y Tierra Bomba y otras haciendas de la Costa Caribe ${ }^{6}$}

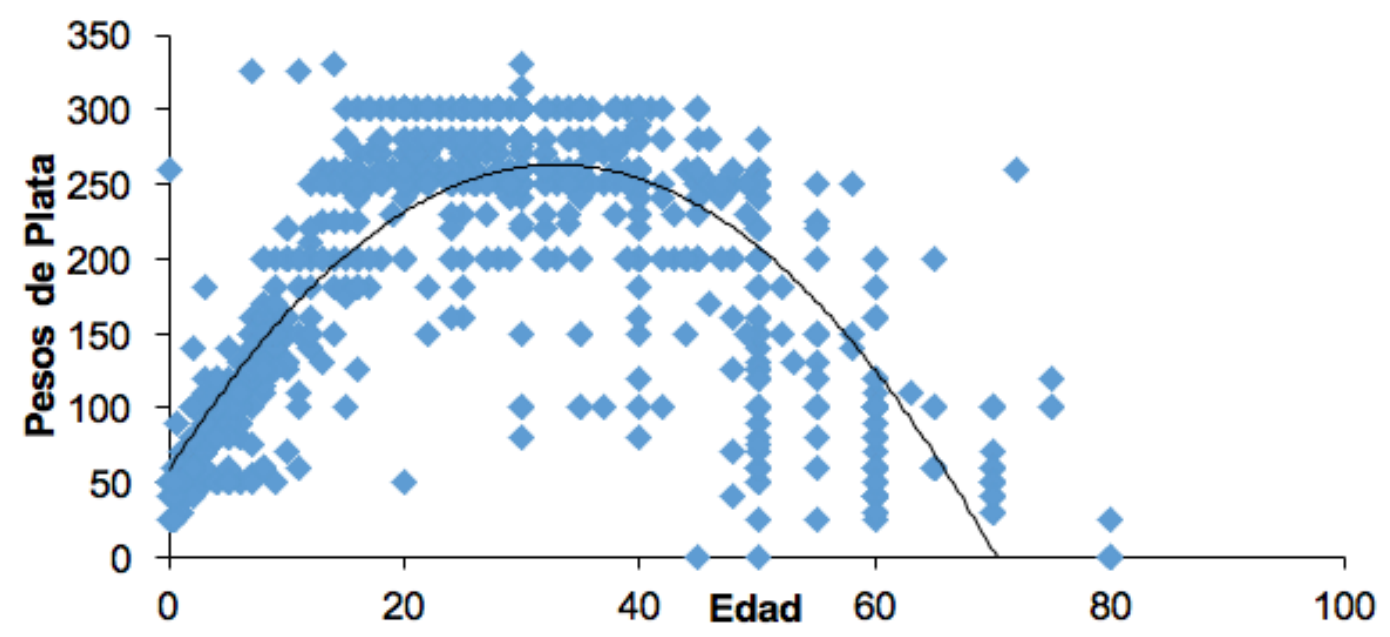

Fuente: elaboración de los autores con datos de Archivo General de la Nación, Sección Colonia, Temporalidades, Tomo 3 , Orden 8, ff. 233-236, 259-260. Sección Colonia, Testamentarias de Bolívar, Tomo 5, Orden 3, ff. 602-610, 612-621, 623-626, 644-645, 682-686, 703-705, 724-725, 732-734. Sección Colonia, Censos Varios Departamentos, ff. 789-790, 799, 800, 804, 817, 823-825, 827,828-832, 838-840. Colombia, Tierras de Bolívar, Tomo I, ff. 137, 924. Tomo II, ff. 636. Tomo III, ff. 396, $399,402$. Tomo V, ff. 452. Tomo VIII, ff. 75. Tomo XII, ff. 655. Colonia, Testamentarias de Bolívar, Tomo 3, Folios: 28-31, 39-41, 45-53, 89-99, 118-127, 130-135, 174-179.

mínimos cuadrados ordinarios y todas las variables independientes resultaron significativas al $1,0 \%$. Con ese modelo se logra explicar el $64,0 \%$ de las variaciones observadas en los precios.

Por último, cabe señalar que el análisis económico del precio de los esclavos muestra que estos se comportan de acuerdo con patrones que revelan una gran racionalidad económica de los propietarios. Es decir, que no es cierto, por lo menos para los esclavos rurales, que se trataba principalmente de un consumo conspicuo, es decir relacionado con afanes de estatus, sino de una inversión destinada a obtener ganancias.

6 Precio $=61,33+12,32$ Edad - 0,18 Edad^2 - 68,01 Enfermo - 6,08 Género + 23,08 Calificación + 11,78 Mina

R2 $=0,64$. Observaciones: 1064

Las haciendas de la Costa Caribe incluidas en esta estimación son: Santa Cruz de Matunilla, Junduras, San Francisco, San Pablo del retiro, Hato de San Marcos, San Andrés de Buena Vista (alias Las Monjas), Señora de la Candelaria, Hato de las Mojarras, Minas de la Soledad, Alto de Puquí, Mina de las Piedras, Estancia del Rayo, Hacienda las Cabezas, Estancia Carinca, Estancia Santa Cruz del Paraíso, y bienes de Don Cayetano Palacios. 


\section{REFERENCIAS}

Abello Vives, A. (2006). Un Caribe sin plantación. San Andrés: Universidad Nacional de Colombia, Sede Caribe : Observatorio del Caribe Colombiano.

Acemoglu, D., García-Jimeno, C., \& Robinson, J. (2012). Finding Eldorado: Slavery and longrun development in Colombia. Journal of Comparative Economics, Elsevier, 40(4), 534-564. https://economics.mit.edu/files/10401

Bergad, L. W. (1987). Slave Prices in Cuba, 1840-1875. The Hispanic American Historical Review, 631-655. https://www.jstor.org/stable/2516047?seq=1\#metadata_info_tab_contents.

Chenny, S., St-Amour, P., \& Vencatachellum, D. (2003). Slave Prices from Succession and Bankruptcy Sales in Mauritius. CIRPÉE. https://www.sciencedirect.com/science/article/pii/ S001449830300041X

Coleman, A., \& Hutchinson, W. (2006). Determinants of slave prices: Louisiana, 1725 TO 1820. Working Paper No. 06-W24. https://ideas.repec.org/p/van/wpaper/0624.html

Conrad, A., \& Meyer, J. (1958). The Economics of Slavery in the Ante Bellum South. Journal of Political Economy, 95-130. https://www.jstor.org/stable/1827270?seq=1\#metadata_info_ tab_contents

Cussen, C., Llorca-Jaña, M., \& Droller, F. (2016). The dynamics and determinants of slave prices in an urban setting: Santiago de Chile, 1773-1822. Revista de Historia Económica / Journal of Iberian and Latin American Economic History, 34(3), 449-477. https://econpapers.repec.org/ article/cupreveco/v_3a34_3ay_3a2016_3ai_3a03_3ap_3a449-477_5fo0.htm

Fogel, R., \& Engerman, S. (1974). Time on the Cross, vol. 1, The Economics of American Negro Slavery; Vol. 2, Evidence and Methods-A Supplement, Little, Brown (1971).

Kotlikoff, L. (1978). The structure of slave prices in New Orleans, 1804 to 1862. University of California at Los Angeles. http://www.piketty.pse.ens.fr/files/Kotlikoff1979.pdf

Meisel, A. (2016, Noviembre). Cartagena de Indias y su tierra adentro a fines del siglo XVIII: Un análisis demoGráfico. Cuadernos de historia económica y empresarial, CEER, Banco de la Republica(42). http://www.banrep.gov.co/es/chee-42

Moreno, M., Klein, H., \& Engerman, S. (1983). The level and structure of slave prices on Cuban Plantations in the Mid-NineteenthCentury: Some comparative perspectives. The American Historical Review, 1201-1218. https://www.jstor.org/stable/1904889?seq=1\#metadata_ info_tab_contents

Newland, C., \& San Segundo, M. (1996). Human capital and other determinants of the price life cycle of a slave: Peru and La Plata in the Eighteenth Century. The Journal of Economic History, 694-701. https://www.cambridge.org/core/journals/revista-de-historia-economica-journal-of-iberian-and-latin-american-economic-history/article/dynamics-and-determinantsof-slave-prices-in-an-urban-setting-santiago-de-chile-c-17731822/7375E2635EC2692DF25E9A39365756B8

Phillips, U. (1905). The economic cost of slaveholding in the cotton belt. Political Science Quarterly, 20(2), 257-275. https://www.jstor.org/stable/2140400?seq=1\#metadata_info_tab_contents 
Ramsdell, C. (1929). The natural limits of slavery expansion. The Southwestern Historical Quarterly, 33(2), 91-111. https://www.jstor.org/stable/30235318?seq=1\#metadata_info_tab_ contents

Sharp, W. (1975). The profitability of slavery in the Colombian Chocó, 1680-1810. The Hispanic American Historical Review, 468-495. https://www.jstor.org/stable/2512376?seq=1\#metadata_info_tab_contents

Sutch, R. C. (2018). The Economics Of African American Slavery: The Cliometrics Debate. NBER Working Paper Series, Working Paper 25197. https://ideas.repec.org/p/nbr/nberwo/25197. html

Tovar, H. et ál. (1994). Convocatoria al poder del número. Bogotá: Archivo General de la Nación. 